\title{
An Information Technology Solution to Enable Remote Training and Operation of Instruments with Out-dated Operating Systems
}

\author{
Jennifer Carter ${ }^{1}$, Jeffrey Pigott ${ }^{1}$, Tae Kyong John Kim $^{2}$ and Steven Waring ${ }^{3}$
}

${ }^{1}$ Swagelok Center for Surface Analysis of Materials, Case Western Reserve University, Cleveland, Ohio, United States, ${ }^{2}$ Swagelok Center for Surface Analysis of Materials, Case Western Reserve University, United States, ${ }^{3}$ Client Platforms, University Technology [u]Tech, Case Western Reserve University, United States

2020 was most indelibly marked by the COVID-19 pandemic. It continues to impact all aspects of life, including the normal operations of Core Research Facilities at all institutions. A major concern for microscopy facilities at universities has been how to reimagine training protocols to accommodate social distancing guidelines. Taking advantage of the Internet of Things has enabled most remote work, but a significant challenge to leveraging the network for core research facilities has been the relative life-expectancy of equipment, versus the fast transition of computer operating systems. The life expectancy of microscopy and analytical equipment can span 10-20 years [1]. In the last 15 years, there have been five different Windows operating systems; currently, only one of those operating systems (Windows 10) is maintained and thus compatible with safe network configurations. The solution is to enable information technology (IT) methods of safe network developments to work around the safety features of an unsecured operating system responsible for maintaining equipment. In this paper, we explain a particular solution that the Swagelok Center for Surface Analysis of Materials (SCSAM) at Case Western Reserve University (CWRU) implemented to 1) select the IT solution, and 2) the cost-benefit analysis for selecting which tools to invest the infrastructure.

SCSAM is CWRU's facility for the microcharacterization of materials located within the Case School of Engineering. SCSAM is a multi-user facility offering a suite of instrumentation for materials characterization and surface and near-surface chemical analysis. Under "normal" conditions, each year more than 250 individual researchers (Figure 1) from the academic, non-profit, and commercial sectors recognize the advantages and benefits SCSAM delivers. In 2019, we had a dramatic transition of technical staff resulting in the center needing to stop training new users. Then in the spring of 2020, like everyone else, we shut our doors to all independent user work to limit the spread of COVID-19. Therefore, we have undergone two-years of not training new users.

The goal of this project is to allow simultaneous remote desktop and in-person control of a microscope and all of its support infrastructure. This allows an engineer to remotely oversee and control the instrument, as well as help, keep in line with covid room occupancy limits. We also need to isolate the instrument computers from our campus network due to a lack of support for old operating systems. To keep things isolated we create a private network between the instrument pc and a new intermediate gateway pc that controls access to the network at large. Users create a remote connection to the intermediate PC through standard encrypted protocols such as Microsoft RDP and from there launch a traditional virtual network computing session through the private network. This infrastructure has been applied to four instruments covering a breadth of applications. In March 2021, students will be trained remotely for independent use of an optical microscope, scanning electron microscope with focused ion beam and 
energy dispersive spectroscopy, time-of-flight secondary ion mass spectrometer, and instruments for x-ray photoelectron.

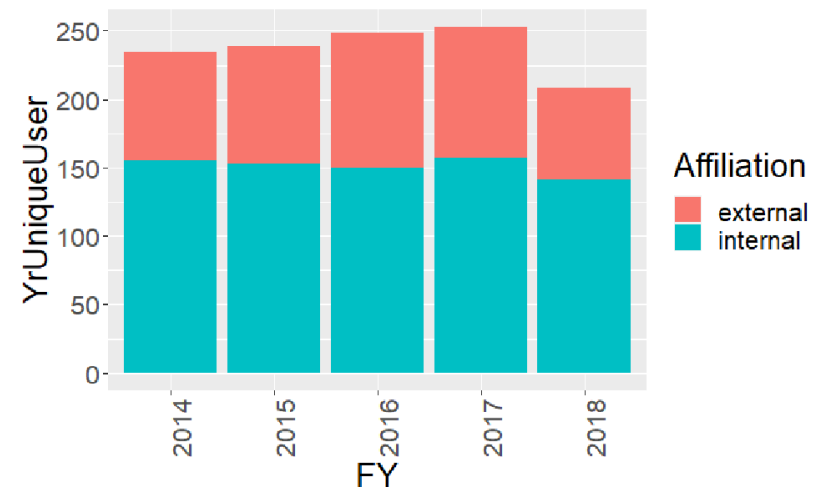

Figure 1. Historical data of the number of unique SCSAM users under normal operating conditions. Two-thirds of the clients are internal (i.e., university students, and researchers), with the remaining users coming from local external, industrial, clients.
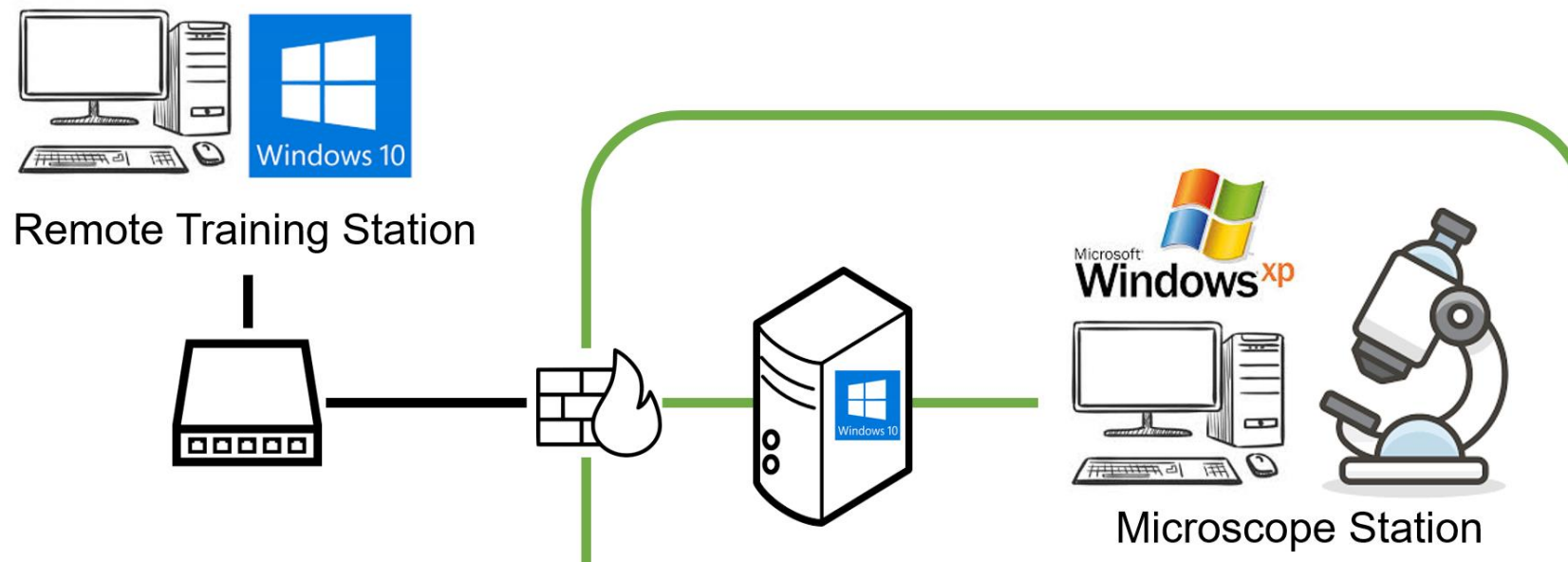

Secured Intranet

Figure 2. Network diagram illustrating the need for a secure firewall for maintaining the integrity of the computing hardware/software that maintains the microscope from malicious external attacks.

\section{References}

G. Owen, "Purchasing an electron microscope? - Considerations and scientific strategies to help in the decision making process," Wiley Analytical Science, 2018. https://analyticalscience.wiley.com/do/10.1002/micro.2610 (accessed Feb. 24, 2021). 